\title{
Stress distribution in restorations with glass fiber and polyetheretherketone intraradicular posts: An in silico analysis
} \author{
William Cunha BRANDT ${ }^{3}$ and Rafael Pino VITTI ${ }^{4}$ \\ ${ }^{1}$ School of Dentistry, São Leopoldo Mandic, Campinas, SP, Brazil \\ ${ }^{2}$ School of Dentistry, Federal University of Santa Catarina, Florianópolis, SC, Brazil \\ ${ }^{3}$ School of Dentistry, Santo Amaro University, São Paulo, SP, Brazil \\ ${ }^{4}$ School of Dentistry, Herminio Ometto University Center, Araras, SP, Brazil \\ Corresponding author, Rafael Pino VITTI; E-mail: rafapvitti@gmail.com
}

Aline Garcia HALLAK ${ }^{1}$, Ricardo Armini CALDAS ${ }^{2}$, Isaias Donizeti SILVA ${ }^{3}$, Milton Edson MIRANDA ${ }^{1}$,

This study evaluated stresses generated at maxillary central incisor (21) root restored with lithium disilicate crown (LDC), and glass fiber (GFP) or polyetheretherketone (PEEK) post. 3D models created by computed tomographic image (i-CAT Cone Beam 3D Dental Imaging System) reproduced maxillary central incisor. Each model had prosthetic LDCs (2.0 mm thick) cemented to GFP or PEEK posts with dual resin cement. Different loads were applied to each model (cervical, incisal, axial, middle). At maximum principal stress, PEEK showed higher stress than GFP in all loads, but with qualitative similarity. At minimum principal stress, PEEK and GFP showed qualitative and quantitative similarities, except axial load. Maxillary central incisor restored with GFP or PEEK and LDC presented, in general, similar stress intensity and distribution for main occlusal loads. Only two of sixteen occlusal loads tested (cervical for maximum principal; axial for minimum principal) showed significant quantitative difference.

Keywords: Finite element analysis, Ceramic, Post and core technique, Polyetheretherketone (PEEK)

\section{INTRODUCTION}

Dentists routinely restore endodontically treated teeth. Root canal preparation may cause the dentin to wear, and lead to weakening of the root from loss of tooth structure, thereby compromising the prognosis of the restorative treatment ${ }^{1,2}$. In most cases, restorative materials (composite resin and ceramics) are bonded to intraradicular retainers (posts) to give the restoration stability and retention. Intraradicular posts that have been incorrectly indicated, or that are poorly used can cause root fracture, and lead to failure of the restoration ${ }^{3-6)}$. The dental surgeon must consider several factors to promote the clinical longevity of these restorations, such as root length and anatomy, retention of the post to the root canal, the elastic modulus of the restorative materials, post diameter and length, and the structure of the remaining tooth ${ }^{1-8}$.

Intraradicular posts are available in a variety of materials, shapes, and sizes. They can be made of metal, glass fiber (GFP), carbon fiber, zirconia, and polyetheretherketone (PEEK). Metal posts have high mechanical strength, but have lost ground to non-metallic materials, since metals are not aesthetic, and can lead to color changes of the remnant tooth caused by corrosion. Furthermore, they have a higher elastic modulus than dentin, thus increasing the chance of root fractures ${ }^{9}$. In contrast, glass fiber, GFP and PEEK posts adhere to the tooth structure, have an elastic modulus more similar to that of the tooth, and are more aesthetic (important

Color figures can be viewed in the online issue, which is available at J-STAGE.

Received Aug 28, 2021: Accepted Nov 24, 2021

doi:10.4012/dmj.2021-245 JOI JST.JSTAGE/dmj/2021-245 physical property for anterior restorations $\mathrm{s}^{5,10-12)}$.

Intraradicular posts can further be classified according to their material, their anatomical shape (cylindrical or conical), and their surface — smooth, serrated or threadable posts have several surface textures. Threadable posts are actively cemented, whereas smooth and serrated varieties are passively cemented $^{5,11)}$. In active cementation, the posts are threaded into the root, enabling a high retention of the post inside the root canal. In passive cementation, the retention of the post in the root is lower than that of active cementation, because it is enabled only by the cement and the irregular surface of the retainer

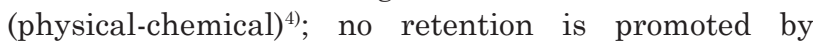
threading (mechanical) ${ }^{4,5,7)}$. Factors needed to achieve adhesion and ward off retention failures include humidity control, protocol of the chemical-mechanical preparation of the retainer, and even the type and quality of the root dentin. It is worth noting that the chemical-mechanical preparation of the canal requires certain irrigants, which contain peroxides, glycols, $\mathrm{NaOCl}$ or EDTA, as well as eugenol-based endodontic cements, all of which have been shown to affect adhesion negatively ${ }^{5,9)}$. Adhesion to the passive retainer is relatively not as strong between cement-retainer as cement-dentin, owing to polymerization shrinkage of the resin cement, and interface failures. This can be dealt with by increasing the adhesion of these types of retainers through effective adaptation, silanization, application of adhesives with 10-methacryloyloxydecyl dihydrogen phosphate (10-MDP) molecules, blasting (alumina or silica) or acid etching. Posts with transverse 
fibers increase adhesion to cement, while vertical fibers improve mechanical resistance to occlusal forces, hence decreasing the chance of root fracture ${ }^{5}$.

The elastic modulus of the restorative materials should be similar to that of the tooth, in order to decrease the intensity of the stresses generated at the toothrestoration interface ${ }^{13,14)}$. The $\mathrm{fit}^{3)}$ and the crown-to-post ratios must be adequate for proper stabilization of the crown-to-post assembly, otherwise running the risk of restoration failure. Several factors may be involved, such as cement degradation, and post fatigue caused by masticatory loads, as well as loosening and displacement of the post within the root canal. The resin cement should promote complete sealing of the irregularities between the post and the root. Thus, the flow of the cementing agent inside the root canal is important to achieve adequate seating of the post, and thus achieve satisfactory stress distribution at the tooth-restoration interface ${ }^{1-2,9,15,16)}$.

The constant development of restorative materials (posts, ceramics, and cements), and the several available restorative protocols for anterior restorations ${ }^{5)}$, along with the disagreement among the studies in the literature, have encouraged new studies to be developed on the topic of anterior restoration procedures. Studies that combine the several variables related to the prognosis of these restorations are important, so that dentists can gain more information to help them make the right choice of restorative materials.

Thus, the aim of this study was to evaluate the stress promoted by a maxillary central incisor restored with GFP or PEEK and a lithium disilicate crown. The hypothesis tested was that different intraradicular retainers would present different stress intensities and distribution patterns.

\section{MATERIALS AND METHODS}

Three-dimensional models for finite element analysis were created based on a computed tomography image (i-CAT Cone Beam 3D Dental Imaging System;
Imaging Sciences International, Norcros, GA, USA) reproducing the maxillary central incisor (21). The images obtained from the CT scan were used as a basis for the computational construction of the anterior maxillary region $(25.0 \mathrm{~mm}$ in height $\times 15.0 \mathrm{~mm}$ in mesiodistal width $\times 15.0 \mathrm{~mm}$ in bucco-palatal depth) constructed from type 3 bone tissue (thin layer of cortical bone $-1.0 \mathrm{~mm}-$ around a trabecular bone), according to its density. Each model consisted of a tooth with a $13.0 \mathrm{~mm}$ root and a $10.0 \mathrm{~mm}$ crown. The prosthetic crowns were represented by lithium disilicate. The experimental groups were modeled using a three-dimensional design software program (SOLIDWORKS ${ }^{\circledR}$ 2013; Dassault Systèmes Solidworks, Greenville, SC, USA), and each model was composed of two $9.0 \mathrm{~mm}$ high intraradicular retainer systems (GFP and PEEK). The post was placed $4.0 \mathrm{~mm}$ above the apical region. The designs were standardized by generating three-dimensional computer models based on the patterns of single tooth incisor restorations.

The lithium disilicate crowns were $2.0 \mathrm{~mm}$ thick. Each crown was cemented to one of two types of posts (GFP and PEEK), with dual-cure resin cement. Both cementation lines were $25.0 \mu \mathrm{m}$ thick. Periodontal ligament had $0.25 \mathrm{~mm}$. The post had cylindrical shape with $2.0 \mathrm{~mm}$ of diameter and thickness (based on FGM, Joinville, SC, Brazil).

All the models were imported into a finite element analysis software (ANSYS Workbench 14 FEA; Swanson Analysis, Canonsburg, PA, USA) for biomechanical analysis. All the materials were considered isotropic, except for the GFP (orthotropic), homogeneous, and linearly elastic; this data were imported into the software program, together with the elastic modulus and the Poisson's ratio values (Table 1). In each model, the cervical, middle, incisal, and axial loads (direction) were applied to the palatal surface (position) with 100 $\mathrm{N}$, simulating the movement and masticatory force that affects the region of the maxillary central incisor teeth. The loading was simulated using nonlinear frictional contact $(0.3)^{17)}$ elements (Table 2).

Table 1 Physical properties of the materials used

\begin{tabular}{|c|c|c|}
\hline Material & Elastic modulus (GPa) & Poisson's coefficient \\
\hline Cortical bone & $13.7^{\mathrm{a}}$ & $0.30^{\mathrm{a}}$ \\
\hline Spongy bone & $1.37^{\mathrm{a}}$ & $0.30^{\mathrm{a}}$ \\
\hline Dentin & $18.6^{b}$ & $0.31^{\mathrm{b}}$ \\
\hline Lithium Disilicate & $68^{\mathrm{c}}$ & $0.24^{\mathrm{c}}$ \\
\hline PEEK & $3.5^{\mathrm{d}}$ & $0.36^{\mathrm{d}}$ \\
\hline GFP & $30^{\mathrm{d}}$ & $0.33^{\mathrm{d}}$ \\
\hline Resin Cement & $7.7^{\mathrm{d}}$ & $0.33^{\mathrm{d}}$ \\
\hline Gutta-percha & $0.00069^{b}$ & $0.45^{\mathrm{b}}$ \\
\hline
\end{tabular}

${ }^{a}$ Çaglar et al., 2011; ${ }^{\mathrm{b}}$ Ko et al., 1992; c Ereifej et al., 2011; ${ }^{\mathrm{d}}$ manufacturer 
Table 2 Number of nodes and elements for each intraradicular retainer

\begin{tabular}{lcc}
\hline Group & Nodes & Elements \\
\hline GFP & 714,861 & 536,753 \\
PEEK & 781,341 & 499,376 \\
\hline
\end{tabular}

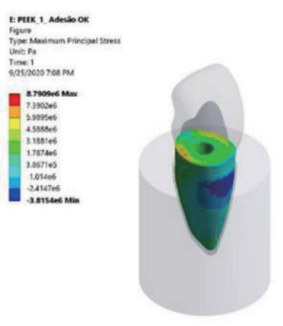

A1
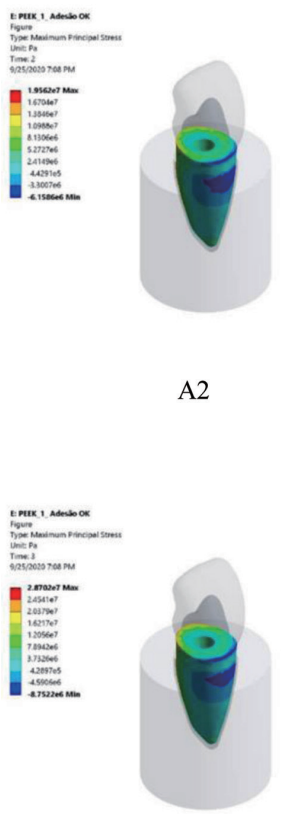

A3

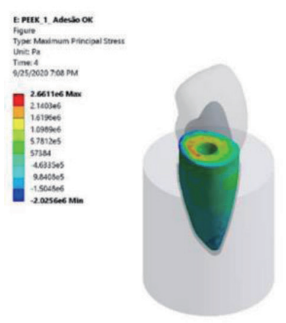

A4

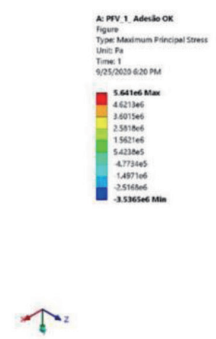

$\uparrow$

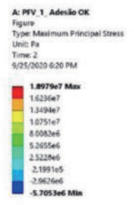

$\uparrow$

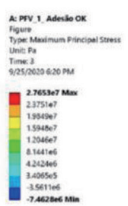

$\uparrow$.

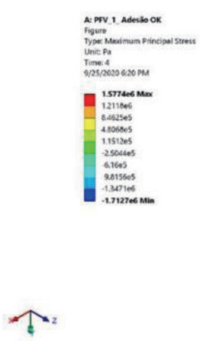

B4

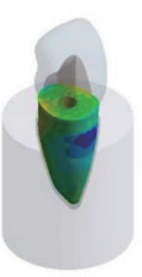

B 1

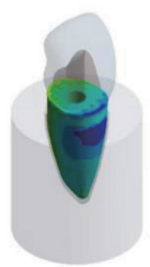

B2

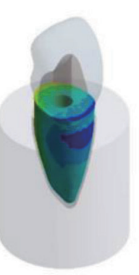

B3

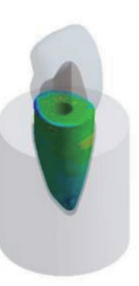

T.2

4

$\uparrow$
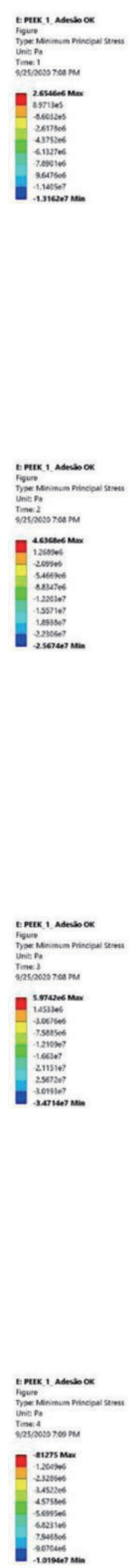

Fig. 1 Maximum principal stress in the roots in (A) PEEK and (B) GFP under (1) cervical, (2) medial, (3) incisal and (4) axial loading.

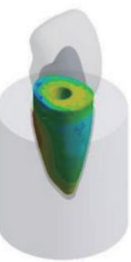

A1

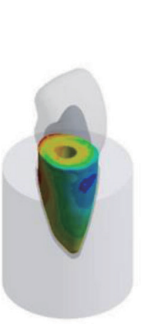

A2

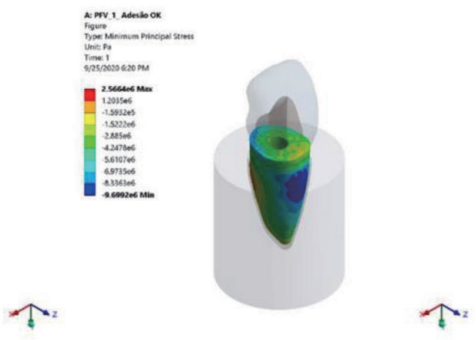

B1

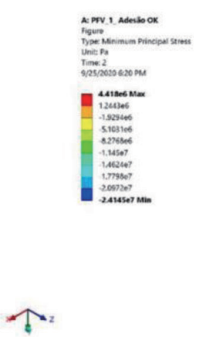

B2
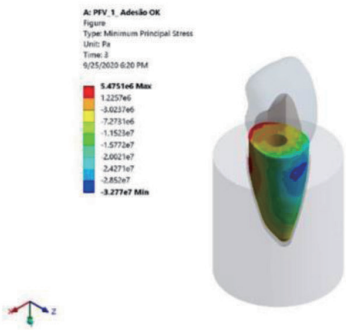

T.

A3

B3
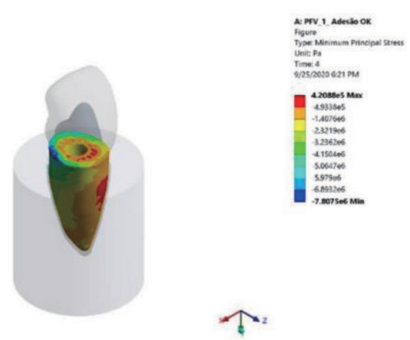

A4

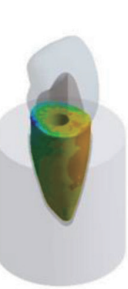

$\uparrow=$

B4

Fig. 2 Minimum principal stress in (A) PEEK and (B) GFP under (1) cervical, (2) medial, (3) incisal and (4) axial loading. 


\section{RESULTS}

All the maximum principal stress analyses showed worse stress results for PEEK than GFP in all loadings tested. In the cervical, incisal, and axial loading, PEEK showed numerically higher maximum principal stress values. Furthermore, from a qualitative point of view, the highest stress values were concentrated in more regions in the tooth restored with PEEK than GFP (Fig. 1; A1-B1, A3-B3 and A4-B4).

At average loading, PEEK and GFP showed similar maximum principal stress values in the quantitative analysis. However, in the other loading conditions, the stresses were concentrated in more regions in the cervical third in the PEEK group than the GFP group (Fig. 1; A2B2). In both groups, when the maximum principal stress was loaded cervically, it was concentrated in the entire cervical third of the root, extending into the periapical region, hence affecting almost all thirds of the lingual root surface (Fig. 1; A1-B1).

In the middle and incisal loadings, the maximum principal stress for PEEK and GFP affected the lingual border of the cervical third, and a large portion of the lingual surface of the root (Fig. 1; A2-B2, A3-B3). In both groups, the maximum principal stress under axial loading was concentrated in the entire cervical third around the intraradicular post (Fig. 1; A4-B4), and this stress extended to the middle third of the root in the GFP (Fig. 1; B4).

PEEK and GFP showed similar minimum principal stress values in all the loadings (Fig. 2; A1-B1, A2-B2 and A3-B3) except the axial load, in which PEEK (8.12 $\mathrm{MPa})$ had almost twice as much stress as GFP (4.20 MPa) (Fig. 2; A4-B4).

Regarding the qualitative analysis, overall, the minimum principal stress was concentrated in the lingual portion of the cervical third of the root, and extended across the lingual surface to the periapical region in the middle and incisal loadings, in both groups (Fig. 2; A2-B2 and A3-B3).

In the cervical loading, the minimum principal stress was concentrated in the entire cervical third and lingual side of the root for PEEK, whereas this stress was concentrated in the vestibular border of the cervical third in addition to the entire lingual side of the root in the GFP group (Fig. 2; A1-B1).

Despite the quantitative difference, when both groups (PEEK and GFP) were loaded axially, they showed similar distribution of the minimum principal stress, which was distributed throughout the cervical third, and which extended across the buccal aspect of the root (Fig. 2; A4-B4).

The results were evaluated qualitatively and quantitatively (Table 3), using the maximum principal (tensile) and minimum principal (compressive) stress values in the root.

\section{DISCUSSION}

The results of the present study show that both materials tested had different intensities of maximum and minimum principal stresses in some loadings; hence, the hypothesis of the study was rejected. However, overall, in most loadings, PEEK and GFP performed similarly in the quantitative and qualitative analyses for the distribution of the maximum and minimum principal stresses. These results can be attributed to the similar difference between the elastic modulus of dentin versus that of GFP and that of PEEK (Table 1).

In the present study, the stress concentration in PEEK and GFP usually started in the cervical third, and extended into a small region of the buccal root surface. In the axial loading, the stress concentration of both groups was around the entire post in the cervical region, but the stress in GFP also dissipated along the tooth axis, up to the middle third of the root. In the maximum principal stress analysis, PEEK showed higher stresses than GFP (Fig. 1), whereas, in the minimum principal stress analysis, PEEK showed almost twice as much stress as GFP during axial loading and generated greater stress in the root (Fig. 2).

The elastic modulus is an intrinsic property of a material, and results from stress and strain. This modulus is influenced by the chemical composition and structure of a material. The ideal material for making root posts would be one with an elastic modulus closest to

Table 3 Maximum and minimum principal stress values for each loading region (cervical, mid, incisal and axial) and each post (PEEK and GFP)

\begin{tabular}{llcc}
\hline & Load & PEEK (MPa) & GFP (MPa) \\
\hline & Cervical & 8.79 & 5.64 \\
Maximum & Medium & 1.95 & 1.89 \\
principal stress & Incisal & 2.87 & 2.76 \\
& Axial & 2.66 & 1.57 \\
& Cervical & 2.65 & 2.56 \\
Minimum & Medium & 4.63 & 4.41 \\
principal stress & Incisal & 5.97 & 5.47 \\
& Axial & 8.12 & 4.20 \\
\hline
\end{tabular}


that of dentin. The elastic modulus of dentin can range from 10 to $30 \mathrm{GPa}$, according to the structural composition of the dentin (age, anatomical differences) $)^{1-2,5,10,18)}$.

PEEK can present different physical properties depending on its manufacturing process, and on the elements added to its composition, such as carbon fibers, glass, hydroxyapatite, or titanium dioxide. Thus, the 3.6 GPa elastic modulus for PEEK can be improved, and approach that of dentin, i.e. $18 \mathrm{GPa}^{19)}$. The PEEK elastic modulus also varies according to its degree of crystallinity. It is important that the standardization of processes be in place when making PEEK intraradicular retainers, and that its elastic modulus be close to that of dentin, in order for the material to have high mechanical properties, without affecting its adhesion to resin cements $^{5,10)}$.

Another limitation of PEEK is its adhesion. Benli et $a l{ }^{20)}$ observed that the PEEK retainer showed increased roughness after its surface was treated with sulfuric acid, as well as greater tensile strength in comparison with GFP. However, sulfuric acid is extremely corrosive and can cause severe tissue damage. Furthermore, its long-term stabilization is unknown, hence making this acid not indicated for use during clinical procedures. Treatment of retainers with sulfuric acid is unadvisable, unless the treatment can be performed during the manufacturing process of the retainer, and its safety can be ensured by the manufacturer. Other studies with other types of acids, such as hydrochloric or nitric acid, were cited in the same study; however, no changes were observed in the PEEK surface, even in higher concentrations. Thus, the aforementioned authors suggest studies be conducted with other forms of surface treatment for PEEK, such as air abrasion, silica coating, application of adhesives, and lasers.

On the other hand, GFP has an elastic modulus of 30 to $50 \mathrm{GPa}^{5,21)}$. GFPs have longitudinal fibers joined uniformly by composites, thus transferring less stress to the restorative material, and providing high flexural and fatigue strength, and high stiffness. This allows stress distribution to remain homogenous during mastication, because it is dissipated along the tooth axis, hence providing the root remnant with greater protection. The diameter of the GFP also influences the flexural strength, because the smaller the diameter, the greater the flexural strength. This may explain why the result of the GFP stress was more homogeneous, and why the stress extended to the middle third of the root, compared with the PEEK stress. Even though this type of prefabricated retainer (GFP) presents mechanical properties similar to those of dentin, it also has some limitations, such as its adaptability to wide canals, and its need for protocols to reduce adhesion failures, based on studies showing that GFPs may exhibit low bond strength between cement and retainer. For this reason, surface treatments, such as silanization, application of adhesives with 10-MDP, abrasive blasting or etching with acidic substances, and self-etching cements are needed to increase the tensile and shear strength at the interface between cement and retainer ${ }^{3,5,13,22)}$.
Although the results of the present study confirm the hypothesis of the current research, future studies are warranted, particularly considering that dentistry is an area with different clinical situations that call for different methods and results. Furthering the research effort is important to increase the knowledge of dental surgeons, so that they can choose the best restorative treatment, particularly for endodontically treated teeth, and those with major coronary destruction. Some variables, such as different stress parameters, loadings, teeth and ferrules, and comparisons with other posts, would also be worthwhile to research in future studies, in addition to modifications in the PEEK composition.

\section{CONCLUSION}

It can be concluded that maxillary central incisors restored with GFPs or PEEK and lithium disilicate crowns, in general, showed similar stress intensity and distribution during the main occlusal load tested.

\section{REFERENCES}

1) Andrade GS, Tribst JP, Dal Piva AO, Bottino MA, Borges AL, Valandro LF, et al. A study on stress distribution to cement layer and root dentin for post and cores made of CAD/CAM materials with different elasticity modulus in the absence of ferrule. J Clin Exp Dent 2019; 11: e1-e8.

2) Sary SB, Samah MS, Walid AAZ. Effect of restoration technique on resistance to fracture of endodontically treated anterior teeth with flared root canals. J Biomed Res 2019; 33: 131-138.

3) Freitas TL, Vitti RP, Miranda ME, Brandt WC. Effect of glass fiber post adaptation on push-out bond strength to root dentin. Braz Dent J 2019; 30: 350-335.

4) Fráter M, Lassila L, Braunitzer G, Vallittu PK, Garoushi S. Fracture resistance and marginal gap formation of post-core restorations: influence of different fiber-reinforced composites. Clin Oral Investig 2020; 24: 265-276.

5) Fernandes V, Ferraz A, Ferreira S, Carvalho O, Silva F, Miller $\mathrm{P}$, et al. Root intracanal posts on rehabilitation of endodontic treated teeth -A narrative review. RevSALUS 2020; 2: 1927.

6) Nagahara T, Takeda K, Inoue S, Wada K, Shirawachi S, Iwata $\mathrm{T}$, et al. Endodontic approach and periodontal regenerative therapy for a mandibular right central incisor affected by a perforation and cemental tear. Int $\mathrm{J}$ Periodontics Restorative Dent 2021; 41: e205-e212.

7) Nahar R, Mishra SK, Chowdhary R. Evaluation of stress distribution in an endodontically treated tooth restored with four different post systems and two different crowns - A finite element analysis. J Oral Biol Craniofac Res 2020; 10: 719-726.

8) Penteado MM, de Andrade GS, Araujo RM, Borges ALS, Valandro LF, Pereira GKR, et al. Fatigue survival of endodontically treated teeth restored with different fiberreinforced composite resin post strategies versus universal 2-piece fiber post system: An in vitro study. J Prosthet Dent 2021; S0022-3913(21)00284-5.

9) Nasr JKC, Correa MF, Ramires MA, Ditzel AS, Manfron APT. Cast metal versus glass fiber post: Literature review. Rev Gestão Saúde 2020; 22: 101-111.

10) Sugano K, Komada W, Okada D, Miura H. Evaluation of composite resin core with prefabricated polyetheretherketone post on fracture resistance in the case of flared root canals. Dent Mater J 2020; 39: 924-932. 
11) Micovic D, Mayinger F, Bauer S, Roos M, Eichberger M, Stawarczyk B. Is the high-performance thermoplastic polyetheretherketone indicated as a clasp material for removable dental prostheses? Clin Oral Investig 2021; 25: 2859-2866.

12) Zarone F, Di Mauro MI, Ausiello P, Ruggiero G, Sorrentino R. Current status on lithium disilicate and zirconia: A narrative review. BMC Oral Health 2019; 19: 134.

13) Lassila L, Oksanen V, Fráter M, Vallittu PK, Garoushi S. The influence of resin composite with high fiber aspect ratio on fracture resistance of severely damaged bovine incisors. Dent Mater J 2020; 39: 381-388.

14) Ibrahim RO, Al-Zahawi AR, Sabri LA. Mechanical and thermal stress evaluation of PEEK prefabricated post with different head design in endodontically treated tooth: 3Dfinite element analysis. Dent Mater J 2021; 40: 508-518.

15) Mishra L, Khan AS, Velo MMAC, Panda S, Zavattini A, Rizzante FAP, et al. Effects of surface treatments of glass fiber-reinforced post on bond strength to root dentine: a systematic review. Materials (Basel) 2020; 13: 1967.

16) Li X, Kang T, Zhan D, Xie J, Guo L. Biomechanical behavior of endocrowns vs fiber post-core-crown vs cast post-core-crown for the restoration of maxillary central incisors with $1 \mathrm{~mm}$ and $2 \mathrm{~mm}$ ferrule height: A 3D static linear finite element analysis. Medicine (Baltimore) 2020; 99: e22648.

17) Caldas RA, Bacchi A, Barão VAR, Versluis A. Should adhesive debonding be simulated for intra-radicular post stress analyses? Dent Mater 2018; 34: 1331-1341.

18) Libonati A, Di Taranto V, Gallusi G, Montemurro E, Campanella V. CAD/CAM customized glass fiber post and core with digital intraoral impression: A case report. Clin Cosmet Investig Dent 2020; 12: 17-24.

19) Bathala L, Majeti V, Rachuri N, Singh N, Gedela S. The role of polyether ether ketone (PEEK) in dentistry —a review. J Med Life 2019; 12: 5-9.

20) Benli M, Eker Gümüş B, Kahraman Y, Huck O, Özcan M. Surface characterization and bonding properties of milled polyetheretherketone dental posts. Odontology 2020; 108: 596-606.

21) Negreiros WA, Regis RR, Pontes KMF, Silva AM, Silva Júnior FI. Effect of restoration technique on stress distribution in extensively destroyed premolars: A finite element analysis studys. RGO Rev Gaúch Odontol 2017; 65: 20-24.

22) Uctasli S, Boz Y, Sungur S, Vallittu PK, Garoushi S, Lassila L. Influence of post-core and crown type on the fracture resistance of incisors submitted to quasistatic loading. Polymers (Basel) 2021; 13: 1130. 